\title{
Planar scintigraphy in assessment of mandibular asymmetry: Unilateral condylar hyperplasia vs asymmetric mandibular hyperplasia
}

\author{
Lo John ${ }^{1 *}$, Yau Yat Yin ${ }^{1}$, Yeung Wai Chow David ${ }^{2}$, Cheung Lim Kwong ${ }^{1}$ \\ ${ }^{1}$ Discipline of Oral and Maxillofacial Surgery, Faculty of Dentistry, the University of Hong Kong, Hong Kong, China \\ ${ }^{2}$ Department of Diagnostic Imaging, Hong Kong Adventist Hospital, Hong Kong, China \\ Email: juno2999@gmail.com
}

Received 2 November 2012; revised 5 December 2012; accepted 17 December 2012

\begin{abstract}
Objectives: To 1) explore the planar scintigraphic findings in asymmetry patients caused by unilateral condylar hyperplasia (UCH) or asymmetric mandibular hyperplasia (AMH); 2) develop a local agecondylar activity reference norm; and 3) check the accuracy of the new norm and compare with other analytical methods. Material and Method: Chinese patients with mandibular asymmetry and scintigraphic findings available were recruited. Clinical, radiographic records and scintigraphic condylar activity ratio (CAR) were studied. Regression analysis was performed to quantify the relationship between age and CAR to develop a new norm. The sensitivity of condylar activity assessment using a) traditional norm; b) new norm; and c) percentile difference was compared. In patients with serial data available, longitudinal analysis of the scintigraphic changes were checked. Result: 109 patients were eligible for the study. Significant difference in CAR was noted between UCH and $A M H$ patients. Linear relationship was observed between age and CAR. A new norm of the age-CAR was established, which showed improved sensitivity in condylar activity prediction in UCH and AMH when compared with traditional norm. Relative to percentile difference, the sensitivity of new norm was lower in AMH but not in UCH patients. Serial analysis revealed gradual decline in CAR with minimal change in percentile difference. Conclusion and Clinical Relevance: There is difference in scintigraphic condylar activity between UCH and AMH patients. A norm of age-CAR relationship was established, which showed improved sensitivity in condylar activity prediction in UCH patients. Further study is required to confirm the role of scintigraphy in AMH.
\end{abstract}

${ }^{*}$ Corresponding author.
Keywords: Scintigraphy; Bone Scan; Facial Asymmetry; Mandibular Asymmetry; Unilateral Condylar Hyperplasia

\section{INTRODUCTION}

Mandibular asymmetry is one of the most common dentofacial deformities among patients seeking treatment [1-3]. The clinical presentation involves deviation of the chin point, canting of the occlusion or the mandibular angle. It is usually the consequence of uneven growth of individual mandibular halves, caused by differential condylar activity. Among all the causes, unilateral condylar hyperplasia (UCH) is regarded as the most common $[4,5]$. A wide diversity of clinical presentation of $\mathrm{UCH}$ has been observed, which can be classified under 3 categories: 1) hemimandibular hyperplasia ( $\mathrm{HH}) ; 2$ ) hemimandibular elongation (HE); and 3) hybrid form which is a combination of $\mathrm{HH}$ and $\mathrm{HE}$ [6]. In addition, patients with mandibular prognathism and asymmetry is believed to be affected by uneven hyperactivity in the growth centers in both mandibular condyles, can be regarded as bilateral hemimandibular elongation or hyperplasia [7], or simply termed as asymmetric man- dibular hyperplasia (AMH).

Patients with mandibular asymmetry usually report the commencement during the onset of puberty, and further aggravation for a period of time after the cessation of growth. Precise estimation of condylar activity plays an important role in surgical planning, as the treatment can be ranged from 1) condylectomy of the affected condyle in active asymmetry; to 2) orthognathic surgery in inactive asymmetry. The assessment can be performed either indirectly from serial comparison of clinical or radiographic records, or directly using skeletal scintigraphy (radionuclide bone scan). Serial assessment using study casts or radiographs appears to be the golden standard, but is time-consuming [8].

Bone scans using technetium 99-methylene bispho- 
sphonate has shown to be effective in direct assessment of the condylar activity [9-11]. Condylar hyperactivity as revealed by rising scintigraphic signal intensity can be associated with growth, neoplastic change, active inflammation and remodeling. The scanning technique includes 2-dimensional planar and 3-dimensional SPECT images. Quantitative analysis is generally preferred nowadays since the first study in 1984, as it allows objective comparison of the activity between the normal and abnormal condyles with higher accuracy [12]. Currently there are 2 methods for the quantitation: 1) indirect assessment using condylar activity ratio (CAR) and 2) direct comparison of both condyles using condylar activity percentage (CA\%). CAR is a ratio of the skeletal activity of the condyle, as revealed by the measured isotope counts/pixel in bone scan, with that of a skeletal reference point with which no further growth activity is assumed. Examples of the skeletal reference include the 4th lumbar vertebrate and clivus. On the other hand, CA\% is calculated using the following equation: [counts/pixel in the condyle/ (sum of counts/pixel in both left and right condyles) $\times$ $100 \%]$.

Using CAR, a linear decrease in skeletal activity along the age was observed in non-asymmetic patients, and a norm of age-CAR was established to assess the condylar activity in patients with mandibular asymmetry [8,12-14]. For the CA\%, it was noted that the normal varia- tion between right and left condyles in patients without any asymmetry was less than $10 \%$ [15]. Subsequent studies in UCH patients always used CA\% with variation more than $10 \%$ as reference of identification of active condyle [16-18]. The role of CAR in assessment of condylar activity in mandibular asymmetry were seldom discussed.

In Hong Kong, condylar activity was assessed in patients with UCH or AMH using planar bone scan from 1989 to 2007, and SPECT since 2007. Using both CAR and CA\% as assessment of condylar activity, it was surprising that the use of age-CAR with reference norms from previous study yielded poor accuracy. On the other hand, in patients with initial active mandibular asymmetry, it is not uncommon to observe a serial reduction of CAR when the condylar activity ceased, while the change in CA\% was minimal. Whether the above observation was due to the difference in race, type of asymmetry, or potential confounding factors such as temporomandibular joint (TMJ) dysfunction or occlusal change induced by orthodontic treatment was poorly understood.

Despite the relatively lower accuracy due to the lack of precise anatomic location of the region of interest, planar bone scan showed a strong correlation with SPECT $[5,9]$. With abundant planar bone scan data available, it was appropriate to conduct a retrospective audit of the scintigraphic findings in patients with mandibular asymmetry caused by UCH/AMH. In particular, the age-CAR relationship in our Hong Kong Chinese sample, and the sensitivity of analysis using age-CAR and CA\% would be evaluated. In addition, any association between scintigraphy and patients' 1) types of mandibular asymmetry, and potential cofounding factors such as 2) TMJ dysfunction and 3) orthodontic treatment, would be assessed as well. It was believed that the findings in this study could be applicable to any future study on assessment of mandibular asymmetry using SPECT.

\section{PATIENTS AND METHOD}

From 1989 to 2007, Chinese patients with facial asymmetry attended to the clinic of Oral and Maxillofacial Surgery, Prince Philip Dental Hospital would be assessed. Patients' records were retrieved for preliminary screening. Patients presented with asymmetry due to increased condylar activity, and having skeletal scintigraphic findings available, were recruited in this study. Patients with 1) asymmetry due to resorptive/pathological changes on condyle; 2) syndromal deformity; and 3) patients with incomplete treatment record to confirm the status and stability of asymmetry, were excluded from this study.

Recruited patients' clinical records were checked for: 1) skeletal diagnosis of asymmetry; 2) any relevant history of lumbar injuries or TMJ pain, and 3) any skeletal relapse following surgical treatment.

For planar scintigraphy, patients were required to receive an intravenous dosage of 20 - 25 mCi Technetium-99 m-methylene diphosphonate 2 - 4 hours before taking static planar images using gamma camera. The planar images included: 1) the anterior and lateral views of mandibular condyles; and 2) posterior view of the 4th lumbar spine (L4). Quantitative analysis was made on a $6 \times 6$ pixel regions of interest placed over both condyles and the 4th lumbar vertebrate (L4). Average counts per pixel for each mandibular condyle and L4 were measured, with background noise corrected. The activity of condyle was expressed as condylar activity ratio (CAR), which was the ratio of counts per pixel of the mandibular condyle to that of L4.

A baseline condylar scintigraphy was routinely prescribed when patients came for initial consultation of their mandibular asymmetry. When the status of asymmetry was either active or uncertain, patients would be put under regular monitoring of the condylar activity, as they were usually unwilling to receive condylectomy performed via extra-oral approach. Surgical treatment would be performed when there was no sign of further progression of the asymmetry, except hemimandibular hyperplasia. The serial condylar activity assessment usually involved clinical examination, dental casts and cephalometric analysis. Inactive asymmetry was defined as no shift in lower dental midline and chin point from pre- 
vious clinical record of 1 year or longer, or no obvious radiographic change from serial tracing. The CAR taken within 1-month of the clinical/radiographic record showing non-growing status was then defined as the final condyle activity ratio (CARf).

The patients' gender, type of asymmetry, CARf of both the affected and normal condyles, and the corresponding age during scintigraphy were recorded. Any history of TMJ clicking or pain, or any concurrent orthodontic treatment happened within one month of having bone scan, were recorded too. Panoramic radiographs would also be checked to rule out any resorptive/pathological disease. This assessment was performed by superimposition of the hand tracings on left and right sides of mandibular ramus and condyle as previously described by Pruzansky [19]. Any obvious difference between the left and right condylar head and process, in terms of height, width, and morphology will also be recorded. When patients had more than one bone scan available, individual CAR would be recorded for assessment of serial changes. For checking planar scintigraphy's sensitivity, the assessment parameters would be tested: 1) ageCAR ratio established from previous studies (Kaban et al. 1995); and 2) CA\% of left and right condyle (Pogrel, 1985).

\section{Statistical Analysis}

The CARf and the corresponding ages were chosen for analysis. Unpaired t-test was chosen to test the association between CARf and 1) gender; 2) age-group; 3) different type of mandibular asymmetry; and 4) effect of TMJ pain or concurrent orthodontic treatment. In addition, the mean and standard deviation (SD) of CARf on both the affected and normal condyles were calculated in each individual age, to allow further investigation of the correlation between condylar activity and age. Comparison of the sensitivity using different parameters was performed using Fisher's exact test. All the analysis was performed using Microsoft Excel 2008 and 2011 for Mac (Microsoft, USA) and Instat3 (Graphpad Software, USA). Statistical significance for the analysis will be set at the level of $\mathrm{p}<0.05$.

\section{RESULTS}

From 1989 to 2007, 293 Chinese patients had planar skeletal scintigraphy performed. Among them, 109 patients were eligible for the analysis. The age ranged from the $14-47$ (mean $=24.41, \mathrm{SD}=7.24)$. The male to female ratio was $47: 62$, with no significant difference (p-value $>0.5$ ) in CARf between the male and female groups. The mean CAR on the affected and normal condyle was 0.95 and 0.86 . The CA\% on the affected and normal condyle was 53 and 47. From the clinical and radiographic records, there was no 1) history of lumbar injury or deformity or 2) sign of skeletal relapse within 2 years after surgery. There were 23 patients showing sign and symptoms of TMJ disorder, and 20 patients having orthodontic treatment during the time of bone scan. Age and gender matched comparison did not reveal any significant difference in CAR or CA\% (p-value $>0.5$ ).

\subsection{Different Forms of Asymmetry}

There were 75 patients showing UCH and 34 patients presenting AMH. Among the UCH patients, 9 were diagnosed with HE, 6 with $\mathrm{HH}$, and the remaining 60 showing hybrid form. For the 6 patients with $\mathrm{HH}$, there was an obvious increase in both CAR (mean $=1.33, \mathrm{SD}=0.54$ ) and CA\% (mean $=61 \%$ ) on the affected condyles as they are still active. Intragroup analysis on patients with stable asymmetry revealed significant difference between the affected side and normal side in UCH group only (Table 1). There was significant difference in CA\% between the hyperactive and normal condyle in UCH group (p-value < 0.01 ), but not in AMH group (p-value $>0.05$ ). The mean percentile difference in UCH group was $6 \%$ whereas the mean difference in UCH group was $2 \%$ only.

The association between different forms of asymmetry and the radiographic findings using superimposition of hand tracing from panoramic radiographs were investigated (Table 2). In UCH group, most of the patients (81.12\%) demonstrated an obvious difference in condylar height. In AMH group, majority (58.82\%) actually showed no obvious difference between the hyperactive and contralateral condyles. Further analysis using Fisher's exact test revealed the number of patients in bilateral group with no obvious difference in radiographic features was significantly higher than that of unilateral group (p-value $<0.001$ ).

\subsection{Correlation of Stable Condylar Activity Ratios and Age}

This correlation analysis involved 1) hyperactive condyles in UCH group and AMH group, and 2) normal condyles in the UCH group only. The mean values of CAR were calculated in individual age and displayed as a scatter plot (Figure 1(a)). A linear relationship was noted between the age and the mean values of CAR of both hyperactive $(\mathrm{R}=0.71)$ and normal $(\mathrm{R}=0.72)$ condyles. For the SD of CAR in individual age, a linear relationship was observed in the normal condyle group $(\mathrm{R}=0.62)$ but not in the hyperactive group $(\mathrm{R}<0.1)$. Hence it is feasible to combine the mean CAR of normal condyle and 1 SD to establish an upper range of CAR. The 3 linear relationships between the CAR and ages were shown in Figure 1(b).

It is noteworthy that the mean final CAR of the 
Table 1. CAR of unilateral condylar hyperactivity and bilateral asymmetry condylar hyperactivity groups.

\begin{tabular}{ccccc}
\hline & \multicolumn{2}{c}{ Unilateral condylar hyperplasia } & \multicolumn{2}{c}{ Asymmetric mandibular hyperplasia } \\
\cline { 2 - 5 } & Hyperactive side & Normal Side & Hyperactive side & Contralateral side \\
\hline $\begin{array}{c}\text { Mean condylar activity ratio } \\
\text { (standard deviation) }\end{array}$ & $0.95(0.23)$ & ${ }^{*} 0.84(0.20)$ & $1.00(0.22)$ & $0.95(0.22)$ \\
$\begin{array}{c}\text { Mean CA\% (standard } \\
\text { deviation) }\end{array}$ & $0.53(0.039)$ & $0.47(0.039)$ & $0.51(0.041)$ & $0.49(0.041)$ \\
$\begin{array}{c}\text { Significant intra-group } \\
\text { difference? (p-value) }\end{array}$ & Yes $(\mathrm{p}<0.01)$ & No $(\mathrm{p}=0.069)$ \\
\hline
\end{tabular}

Table 2. Difference between UCH and AMH patients in terms of radiographic presentation.

\begin{tabular}{cccc}
\hline $\begin{array}{c}\text { Difference between the hyperactive and } \\
\text { contralateral/normal condyle }\end{array}$ & UCH (n = 69) & AMH (n = 34) & $\begin{array}{c}\text { p-value using Fisher's } \\
\text { exact test }\end{array}$ \\
\hline Minimal/no difference (\%) & 5.80 & 58.82 & $<0.0001$ \\
Difference involving condylar height (\%) & 81.12 & 32.35 & $<0.0001$ \\
Difference involving the width of condylar head (\%) & 17.39 & 8.82 & 0.3744 \\
Difference involving condylar morphology (\%) & 5.80 & 2.94 & 0.6818 \\
Difference involving angulation of condylar process (\%) & 10.14 & & 0.2657 \\
\hline
\end{tabular}

hyperactive condyle was in the range between the mean and upper range of the CAR of normal condyle. The range of norm values from this study was listed in Table 3 in which a linear drop of around $0.02-0.03$ CAR (approximate $2 \%$ - 3\% of baseline CAR) per year was observed.

\subsection{Sensitivity in Assessment of Condylar Hyperactivity}

The sensitivity of condylar activity in patients with inactive asymmetry was evaluated based on the final CAR.

Three assessment parameters would be tested: 1) the age-CAR ratio established from previous study (Kaban et al. 1995); 2) the new age-CAR ratio developed in this retrospective study; and 3 ) difference in condylar activity percentage (CA\%) with variation less than $10 \%$ as normal (Pogrel, 1985).

The findings (Table 4) revealed lowest sensitivity of condylar activity assessment if the age-CAR ratio established from previous reported data (Kaban et al. 1995). Using the new age-CAR ratio, the sensitivity was significantly improved (p-value $<0.0001$ ). Nevertheless, the sensitivity on affected condyle using new age-CAR ratio was significantly lower than that of normal condyle (pvalue $=0.034)$ and that using CA\% (p-value $=0.0499)$.

Further analysis was made by sub-dividing the sample into UCH and AMH groups. Improvement in sensitivity on affected condyle using new age-CAR ratio was noted in UCH group, which revealed no significant difference (p-value $=0.46$ ) compared to that using CA\%. However, a drop of sensitivity on affected condyle using the new
age-CAR ratio was further noted noted in bilateral group, which was significantly lower than the sensitivity using CA\% (p-value $=0.034)$. To remove the bias due to unbalanced sample size, an age and gender matched comparison was made between the unilateral and bilateral asymmetric groups. It seems that sensitivity in affected condyle using age-CAR ratio was almost identical to that using CA\% in UCH group (p-value $=1.00)$. The use of age-CAR ratio in prediction of condylar activity appeared to be sensitive in 1) hyperactive condyle in patients with unilateral condylar hyperactivity only; and 2) normal/ contralateral condyle in both unilateral and bilateral condylar hyperactivity. On the other hand, use of CA\% was sensitive in predicting of condylar activity in both unilateral and bilateral condylar hyperactivity.

\subsection{Serial Change of Scintigraphy}

There were 38 patients having serial planar bone scan data available (18 with 2 serial scans, 20 with 3 or more serial scans). From the 18 patients (9-unilateral group, 9-bilateral group) with 2 serial scintigraphy data, both clinical and radiographic assessment did not show any obvious serial change in mandibular asymmetry and hence the condyle could be considered as inactive in all cases. On the other hand, in the 20 patients with 3 or more scintigraphy data, serial clinical and radiographic comparison could only reveal initial active asymmetry in 9 patients only, and the remaining 11 patients did not show any sign of further progression of asymmetry during serial analysis.

All patients with initial active asymmetry showed 


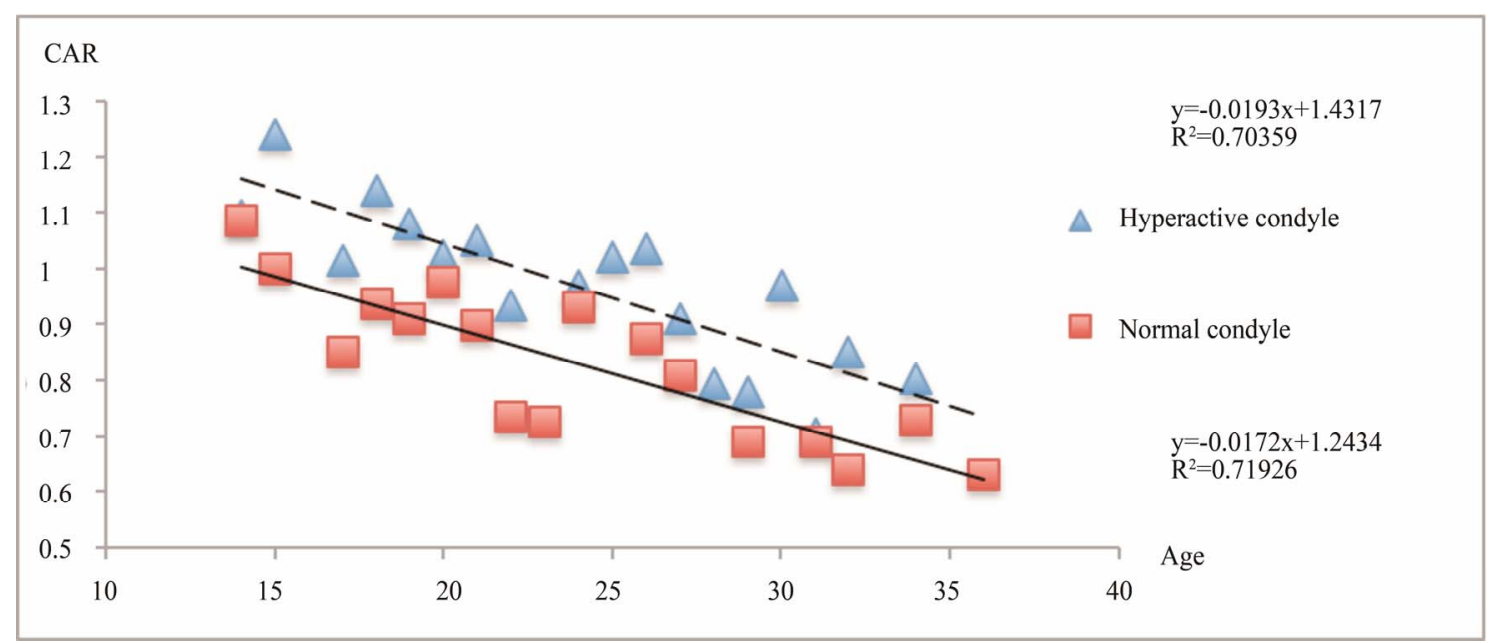

(a)

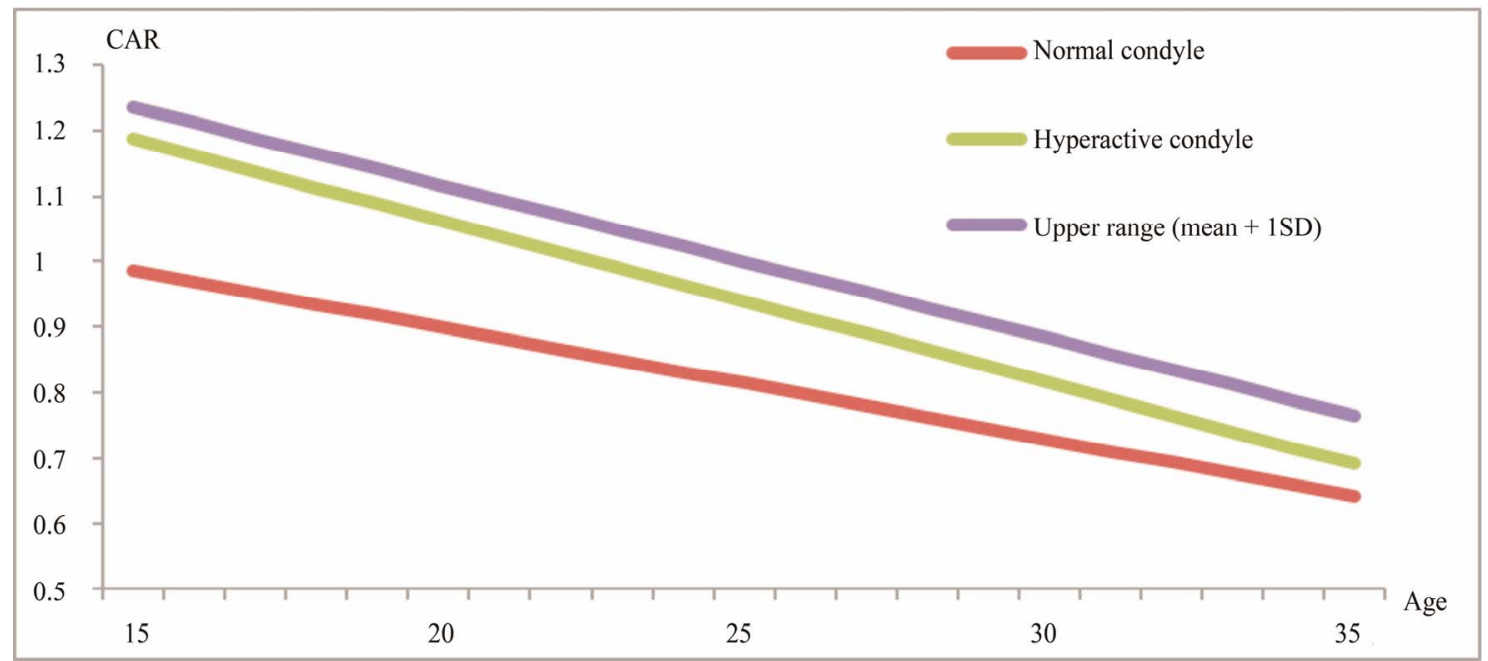

(b)

Figure 1. The change of condylar activity along the age shown; (a) scattering plot of the mean CAR at individual age; and (b) estimated linear change based on the regression analysis of the mean, standard deviation (SD) of CAR and age; (a) Scattering plot of the mean CAR at individual age showing a linear decline in condylar activity in both hyperactive and normal condyle; (b) The estimated change of CAR along the age in 1) normal condyle; 2) affected (hyperactive) condyle; and 3) upper range of the normal condyle (mean CAR + 1 standard deviation (SD)).

Table 3. Predicted variation of condylar activity ratio using mean and mean +1 SD of CAR of the normal condyle in the unilateral condylar hyperactivity group.

\begin{tabular}{cc}
\hline Age range & Condylar activity ratio \\
\hline $15-20$ & $1.11-1.23$ \\
$20--25$ & $0.99-1.10$ \\
$25-30$ & $0.88-0.98$ \\
\hline
\end{tabular}

reduction in CAR when it became inactive. In patients with inactive asymmetry, serial reduction of CAR was noted in $75 \%$ and $30.77 \%$ of patients in UCH and AMH groups respectively (Table 5). The mean serial change of CAR was 0.18 per year, with no significant difference between active and inactive groups, or between $\mathrm{UCH}$ and AMH groups (p-value ranged from 0.53 - 0.96). The serial change in CA\% was significantly smaller than CAR (p-value < 0.00001) with high variation in both active and inactive groups.

The accuracy of using the new age-CAR ratio and the CA\% (with $>10 \%$ difference as active condyle) in serial assessment of those patients with initial active asymmetry was also checked (Table 6). Although longitudinal reduction of CAR is noted, it was not uncommon that in some cases the CAR was still higher than the predicted value from the new age-CAR ratio. On the other hand, the use of CA\% with percentile difference $<10 \%$ as normal, did not show any additional advantage in serial assessment of patient with active asymmetry. In AMH 
Table 4. Comparison of sensitivity in assessment of condy- lar hyperactivity using CA\% with the difference $<10 \%$ being normal (Pogrel 1985) and age-CAR ratio developed from this study.

\begin{tabular}{|c|c|c|c|c|c|}
\hline \multirow[t]{2}{*}{$\begin{array}{l}\text { Assessment } \\
\text { parameter }\end{array}$} & \multicolumn{2}{|c|}{$\begin{array}{l}\text { Age-CAR ratio } \\
\text { (previous data) }\end{array}$} & \multicolumn{2}{|c|}{$\begin{array}{l}\text { Age-CAR ratio } \\
\text { (from this study) }\end{array}$} & \multirow{2}{*}{$\begin{array}{l}\text { CA\% } \\
\mathbf{5 \% - 5 5 \% )}\end{array}$} \\
\hline & Affected & \multicolumn{2}{|c|}{ Whole group $(\mathrm{n}=103)$} & Normal & \\
\hline $\begin{array}{l}\text { Sensitivity } \\
\text { (\%) }\end{array}$ & 29.13 & 44.67 & 62.14 & 76.70 & 75.73 \\
\hline $\begin{array}{c}\text { p-value } \\
\text { (compared } \\
\text { with CA\%) }\end{array}$ & $<0.0001$ & $<0.0001$ & 0.0499 & 1.000 & N/A \\
\hline \multicolumn{6}{|c|}{ Unilateral hyperactive group $(n=69)$} \\
\hline $\begin{array}{c}\text { Sensitivity } \\
(\%)\end{array}$ & 26.09 & 46.38 & 65.22 & 79.71 & 72.46 \\
\hline $\begin{array}{c}\text { p-value } \\
\text { (compared } \\
\text { with CA\%) }\end{array}$ & $<0.0001$ & $<0.0001$ & 0.4625 & 0.4250 & N/A \\
\hline \multicolumn{6}{|c|}{ Bilatera asymmetric group $(\mathrm{n}=34)$} \\
\hline $\begin{array}{l}\text { Sensitivity } \\
\text { (\%) }\end{array}$ & 35.29 & 41.18 & 55.88 & 70.59 & 82.35 \\
\hline $\begin{array}{c}\text { p-value } \\
\text { (compared } \\
\text { with CA\%) }\end{array}$ & 0.0002 & 0.0010 & 0.0344 & 0.3917 & N/A \\
\hline
\end{tabular}

Table 5. Longitudinal change of scintigraphy findings in 38 patients with 2 or more bone scans performed.

\begin{tabular}{lcccc}
\hline & \multicolumn{2}{c}{ UCH } & \multicolumn{2}{c}{ AMH } \\
\cline { 2 - 5 } & Active & Inactive & Active & Inactive \\
\hline Type of change in CAR & & & & \\
$\begin{array}{l}\text { 1) Increase (continuous) } \\
\begin{array}{l}\text { 2) Increase followed by } \\
\text { reduction }\end{array}\end{array}$ & 0 & 0 & 0 & 2 \\
$\begin{array}{l}\text { 3) Increase followed by } \\
\text { minimal change }\end{array}$ & 0 & 1 & 3 & 1 \\
$\begin{array}{l}\text { 4) Serial reduction } \\
\text { 5) Minimal change }\end{array}$ & 3 & 12 & 1 & 4 \\
Total & 0 & 0 & 0 & 3 \\
Serial change (CAR) & 0.16 & 0.16 & 0.31 & 0.16 \\
Serial change (CA\%) & 0.027 & 0.032 & 0.019 & 0.023 \\
\hline
\end{tabular}

Table 6. Accuracy of using age-CAR ratio and CA\% (with $>10 \%$ difference between $\mathrm{L} \& \mathrm{R}$ condyle as active) in serial assessment of patient with initial active asymmetry (9 patients).

\begin{tabular}{ccccc}
\hline & \multicolumn{2}{c}{ UCH } & \multicolumn{2}{c}{ AMH } \\
\cline { 2 - 5 } & CAR & CA\% & CAR & CA\% \\
\hline Sensitivity (\%) & 67 & 50 & 50 & 0.00 \\
Specificity (\%) & 75 & 100 & 50 & 75 \\
\hline
\end{tabular}

patients with initial active asymmetry, all of them showed normal CA\% (with $10 \%$ percentile difference) during the first scan. The serial change in majority of the patients was still within normal CA\% as well.

\section{DISCUSSION}

Despite using similar skeletal reference (4 th lumbar vertebrate), the age-CAR relationships developed from this study showed obvious difference with the previous reported normal values based on 90 non-asymmetric subjects in United States with age ranged from 0 - 30 years [14]. The difference could be due to the ethnic factor, which could result in difference in physical activity, dietary calcium intake and the bone mineral content of the lumbar spine [20,21]. In addition, age-CAR normal values developed in this study were based on the normal condyle of patients with unilateral condylar hyperactivity rather than non-asymmetry patients. While there was concern on the stress on the normal condyle due to the hyperactive condyle, the condylar activity should not be permanently disturbed as the shift was not a dramatic process as reported from one animal studies showing transient fluctuation of scintigraphic activity following sudden change in occlusion [22]. On the other hand, the predicted CAR value from 15 - 30 years from this study was closer to another study in Australia [5], which was based on 16 non-asymmetric subjects with age ranged from 10 - 30 years (Table 7). Apart from this retrospective study, this age-CAR relationship was also tested in our on-going prospective study of patients with mandibular asymmetry, with similar sensitivity being noted [23].

While the use of CA\% was well accepted as the parameter in determination of the status of the condylar activity [15,16,18,24], majority of the studies was based on single bone scan data only. So far there was only one clinical study of unilateral condylar hyperactivity patients showing serial SPECT analysis in 4 out of 18 patients [16], which revealed serial reduction of CA\% in all patients without any clear description of the final status of the condylar activity. The findings from our study supported the use of CA\% in cross-sectional assess-

Table 7. Comparison of the predicted age-CAR values from this study with other reported studies.

\begin{tabular}{cccc}
\hline Study & Sample & Age range & CAR range \\
\hline $\begin{array}{c}\text { Kaban et al } \\
1995\end{array}$ & 90 & $15-20$ & $1.10-0.70$ \\
$\begin{array}{c}\text { (United States) } \\
\begin{array}{c}\text { Chan et al 2000 } \\
\text { (Australia) }\end{array}\end{array}$ & 16 & $10-30$ & $0.66-1.34$ \\
$\begin{array}{c}\text { This study } \\
\text { (Hong Kong) }\end{array}$ & 103 & $15-30$ & $0.72-1.23$ \\
\hline
\end{tabular}


ment only in UCH group. Nevertheless it was noteworthy that persistent high CA\% (>10\%) was usually noted in asymmetry patients with obvious difference between affected and contralateral condyles. In longitudinal follow-up of those patients with active asymmetry, CA\% did not offer any additional advantage relative to CAR. Nevertheless, our sample was only consisted of 8 patients' planar data with initially active asymmetry. Further study using SPECT is required to redefine the role of CAR and CA\% in serial analysis of patients with active mandibular asymmetry.

The validity of skeletal scintigraphy as assessment tool had been widely reported in UCH patients, whereas information on AMH was scanty. This study revealed difference in the scintigraphic activity between UCH and $\mathrm{AMH}$, and confirmed hyperactivity in both condyles in AMH with no significant difference in CA\% between the hyperactive and contralateral condyles. AMH patients usually showed obvious mandibular prognathism apart from asymmetry. Unlike UCH, 58\% of AMH patients did not revealed any obvious difference between the mandibular condyles. Although there were numerous studies aimed to explore the relationship between bone scan activity and mandibular condyles, a number of controversies still existed. While earlier study using planar data reported the positive relationship between bone scan activity and the depth of hypertrophic cartilage island in condylar head [25], recent studies based on SPECT data failed to identify any histopathological relationship [26, 27]. In this study, it seems that obvious dimensional difference between the affected and normal condyles tended to give rise a persistent high CA\% in patients with $\mathrm{UCH}$.

Skeletal scintigraphy was regarded as sensitive but not specific. Apart from growth, an increase condylar activity as revealed by bone scan could also be associated with inflammation, neoplasm, remodeling process [28], which could be associated by TMJ dysfunction, trauma or orthodontic treatment. Despite good correlation being reported from other studies [29-31], the finding from our study did not reveal any significant association between TMJ dysfunction and skeletal scintigraphy. It could be due to our sample consisted mainly of patients with clicking which was only identified on clinical examination only. On the other hand, the samples from those studies supporting the correlation were consisted of patients presenting with chief complaint including TMJ pain or osteoarthritis. There were only 3 patients presenting with complaint of pain. While age match comparison did not revealed any

significant difference, all the 3 patients' CAR value was found beyond the norm value obtained from the new age-CAR ratio from this study. Owing to the limited sample size, further study is recommended to check whether that age-CAR ratio could also be considered as one of the assessment parameter in patients with TMJ dysfunction. Regarding the effect of orthodontic treatment, this study did not show any significant association between fixed appliance therapy and scintigraphic activity. In fact, the use of scintigraphy in assessment of orthodontic treatment was mainly in the field of functional appliance therapy [32,33]. As there was animal study reporting the transient change of scintigraphy activity caused by sudden change in occlusion [22], it seems that the gradual shift in occlusion caused by the orthodontic appliance would not induce any permanent change in bone scan.

In conclusion, this retrospective study on 109 patients with mandibular asymmetry showed the difference in terms of planar scintigraphic activity and radiographic feature between unilateral condylar hyperactivity and bilateral asymmetric condylar hyperactivity. A linear relationship between age and condylar activity ratio was observed in both the affected and contralateral condyles, which was different from the previous studies. Using the new age-condylar activity ratio we could achieve better sensitivity in prediction of condylar activity in patients with initial active mandibular asymmetry when compared with condylar activity percentage. Further SPECT study based on age-condylar activity ratio in checking the serial change of condylar activity was recommended.

\section{ACKNOWLEDGEMENTS}

The authors would like to acknowledge Mr. Shadow Yeung for the assistance in statistic analysis.

\section{REFERENCES}

[1] Severt, T.R. and Proffit, W.R. (1997) The prevalence of facial asymmetry in the dentofacial deformities population at the University of North Carolina. International Journal of Adult Orthodontics \& Orthognathic Surgery, 12, 171-176.

[2] Haraguchi, S., Takada, K. and Yasuda, Y. (2002) Facial asymmetry in subjects with skeletal Class III deformity. Angle Orthodontist, 72, 28-35.

[3] Samman, N., Tong, A.C., Cheung, D.L. and Tideman, H. (1992) Analysis of 300 dentofacial deformities in Hong Kong. International Journal of Adult Orthodontics \& Orthognathic Surgery, 7, 181-185.

[4] Kaban, L.B. (2009) Mandibular asymmetry and the fourth dimension. Journal of Craniofacial Surgery, 20, 622-631. doi:10.1097/SCS.0b013e318195249c

[5] Chan, W.L., Carolan, M.G., Fernandes, V.B. and Abbati, D.P. (2000) Planar versus SPET imaging in the assessment of condylar growth. Nuclear Medicine Communications, 21, 285-290.

doi:10.1097/00006231-200003000-00013

[6] Obwegeser, H.L. and Makek, M.S. (1986) Hemimandibular hyperplasia-Hemimandibular elongation. Journal 
of Maxillofacial Surgery, 14, 183-208. doi:10.1016/S0301-0503(86)80290-9

[7] Obwegeser, H.L. (2001) Bilateral hemimandibular elongation. In: Mandibular Growth Anomalies, Springer, New York, 242.

[8] Fahey, F.H., Abramson, Z.R., Padwa, B.L., Zimmerman, R.E., Zurakowski, D., Nissenbaum, M., Kaban, L.B. and Treves, S.T. (2010) Use of (99m)Tc-MDP SPECT for assessment of mandibular growth: Development of normal values. European Journal of Nuclear Medicine and Molecular Imaging, 37, 1002-1010. doi:10.1007/s00259-009-1337-0

[9] Pogrel, M.A., Kopf, J., Dodson, T.B., Hattner, R. and Kaban, L.B. (1995) A comparison of single-photon emission computed tomography and planar imaging for quantitative skeletal scintigraphy of the mandibular condyle. Oral Surgery, Oral Medicine, Oral Pathology, Oral Radiology and Endodontology, 80, 226-231. doi:10.1016/S1079-2104(05)80206-9

[10] Henderson, M.J., Wastie, M.L., Bromige, M., Selwyn, P. and Smith, A. (1990) Technetium-99m bone scintigraphy and mandibular condylar hyperplasia. Clinical Radiology, 41, 411-414. doi:10.1016/S0009-9260(05)80603-8

[11] Saridin, C.P., Raijmakers, P.G., Tuinzing, D.B. and Becking, A.G. (2011) Bone scintigraphy as a diagnostic method in unilateral hyperactivity of the mandibular condyles: A review and meta-analysis of the literature. International Journal of Oral \& Maxillofacial Surgery, 40, 11-17. doi:10.1016/j.ijom.2010.09.015

[12] Cisneros, G.J. and Kaban, L.B. (1984) Computerized skeletal scintigraphy for assessment of mandibular asymmetry. Journal of Oral and Maxillofacial Surgery, 42, 513520. doi:10.1016/0278-2391(84)90010-7

[13] Kaban, L.B., Cisneros, G.J., Heyman, S. and Treves, S. (1982) Assessment of mandibular growth by skeletal scintigraphy. Journal of Oral and Maxillofacial Surgery, 40, 18-22. doi:10.1016/S0278-2391(82)80010-4

[14] Kaban, L.B., Treves, S.T., Pogrel, M.A. and Hattner, R.S. (1995) Skeletal scintigraphy for assessment of mandibular growth and asymmetry. In: S. T. Treves, Ed., Paediatric Nuclear Medicine, Springer, New York.

[15] Pogrel, M.A. (1985) Quantitative assessment of isotope activity in the temporomandibular joint regions as a means of assessing unilateral condylar hypertrophy. Oral Surgery, Oral Medicine, Oral Pathology, 60, 15-17. doi:10.1016/0030-4220(85)90206-3

[16] Hodder, S.C., Rees, J.I., Oliver, T.B., Facey, P.E. and Sugar, A.W. (2000) SPECT bone scintigraphy in the diagnosis and management of mandibular condylar hyperplasia. British Journal of Oral and Maxillofacial Surgery, 38, 87-93. doi:10.1054/bjom.1999.0209

[17] Saridin, C.P., Raijmakers, P.G., Tuinzing, D.B. and Becking, A.G. (2008) Comparison of planar bone scintigraphy and single photon emission computed tomography in patients suspected of having unilateral condylar hyperactivity. Oral Surgery, Oral Medicine, Oral Pathology, Oral Radiology and Endodontology, 106, 426-432. doi:10.1016/j.tripleo.2008.01.028

[18] Saridin, C.P., Raijmakers, P.G., Al Shamma, S., Tuinzing,
D.B. and Becking, A.G. (2009) Comparison of different analytical methods used for analyzing SPECT scans of patients with unilateral condylar hyperactivity. International Journal of Oral \& Maxillofacial Surgery, 38, 942946. doi:10.1016/j.ijom.2009.04.019

[19] Pruzansky, S. (1969) Not all dwarfed mandibles are alike. Birth defects, 1.

[20] Burrows, M., Baxter-Jones, A., Mirwald, R., Macdonald, H. and McKay, H. (2009) Bone mineral accrual across growth in a mixed-ethnic group of children: Are Asian children disadvantaged from an early age? Calcified Tissue International, 84, 366-378. doi:10.1007/s00223-009-9236-8

[21] McKay, H.A., Petit, M.A., Khan, K.M. and Schutz, R.W. (2000) Lifestyle determinants of bone mineral: A comparison between prepubertal Asian- and Caucasian-Canadian boys and girls. Calcified Tissue International, 66, 320-324. doi:10.1007/s002230010067

[22] Yokoyama, M., Atsumi, T., Tsuchiya, M., Koyama, S. and Sasaki, K. (2009) Dynamic changes in bone metabolism in the rat temporomandibular joint after molar extraction using bone scintigraphy. European Journal of Oral Sciences, 117, 374-379. doi:10.1111/j.1600-0722.2009.00635.x

[23] Lo, J., Yau, Y.Y. and Cheung, L.K. (2010) Diagnostic accuracy of planar bone scintigraphy in mandibular asymmetry using a new reference. $X X$ Congress of the European Association for Cranio-Maxillo-Facial Surgery: Bruges.

[24] Kajan, Z.D., Motevasseli, S., Nasab, N.K., Ghanepour, H. and Abbaspur, F. (2006) Assessment of growth activity in the mandibular condyles by single-photon emission computed tomography (SPECT). Australian Orthodontic Journal, 22, 127-130.

[25] Gray, R.J., Sloan, P., Quayle, A.A. and Carter, D.H. (1990) Histopathological and scintigraphic features of condylar hyperplasia. International Journal of Oral \& Maxillofacial Surgery, 19, 65-71. doi:10.1016/S0901-5027(05)80196-1

[26] Villanueva-Alcojol, L., Monje, F. and Gonzalez-Garcia, R. (2011) Hyperplasia of the mandibular condyle: Clinical, histopathologic, and treatment considerations in a series of 36 patients. Journal of Oral and Maxillofacial Surgery, 69, 447-455. doi:10.1016/j.joms.2010.04.025

[27] Saridin, C.P., Raijmakers, P.G., Slootweg, P.J., Tuinzing, D.B., Becking, A.G. and van der Waal, I. (2010) Unilateral condylar hyperactivity: A histopathologic analysis of 47 patients. Journal of Oral and Maxillofacial Surgery, 68, 47-53. doi:10.1016/j.joms.2009.07.033

[28] Obwegeser, H.L. (2001) Scintigraphy, a diagnostic and treatment planning aid. In: Mandibular Growth Anomalies, Springer, New York, 31-33.

[29] Kim, J.H., Kim, Y.K., Kim, S.G., Yun, P.Y., Kim, J.D. and Min, J.H. (2011) Effectiveness of bone scans in the diagnosis of osteoarthritis of the temporomandibular joint. Dentomaxillofacial Radiology.

[30] Coutinho, A., Fenyo-Pereira, M., Dib, L.L. and Lima, E.N. (2006) The role of SPECT/CT with 99mTc-MDP image fusion to diagnose temporomandibular dysfunction. 
Oral Surgery, Oral Medicine, Oral Pathology, Oral Radiology and Endodontology, 101, 224-230.

doi:10.1016/j.tripleo.2005.03.018

[31] Epstein, J.B., Rea, A. and Chahal, O. (2002) The use of bone scintigraphy in temporomandibular joint disorders. Oral Diseases, 8, 47-53.

doi:10.1034/j.1601-0825.2002.10753.X

[32] Guner, D.D., Ozturk, Y. and Sayman, H.B. (2003) Evaluation of the effects of functional orthopaedic treatment on temporomandibular joints with single-photon emission computerized tomography. European Journal of Orthodontics, 25, 9-12. doi:10.1093/ejo/25.1.9

[33] Paulsen, H.U., Rabol, A. and Sorensen, S.S. (1998) Bone scintigraphy of human temporomandibular joints during Herbst treatment: A case report. European Journal of Orthodontics, 20, 369-374. doi:10.1093/ejo/20.4.369 